University of Nebraska - Lincoln

DigitalCommons@University of Nebraska - Lincoln

$11-1-2002$

\title{
Effect of an Electric Field on the Surface Tension of a Dipolar- Quadrupolar Fluid and Its Implication for Sign Preference in Droplet Nucleation
}

\author{
V.B. Warshavsky \\ University of Nebraska-Lincoln \\ Xiao Cheng Zeng \\ University of Nebraska-Lincoln, xzeng1@unl.edu
}

Follow this and additional works at: https://digitalcommons.unl.edu/chemzeng

Part of the Chemistry Commons

Warshavsky, V.B. and Zeng, Xiao Cheng, "Effect of an Electric Field on the Surface Tension of a DipolarQuadrupolar Fluid and Its Implication for Sign Preference in Droplet Nucleation" (2002). Xiao Cheng Zeng Publications. 38.

https://digitalcommons.unl.edu/chemzeng/38

This Article is brought to you for free and open access by the Published Research - Department of Chemistry at DigitalCommons@University of Nebraska - Lincoln. It has been accepted for inclusion in Xiao Cheng Zeng Publications by an authorized administrator of DigitalCommons@University of Nebraska - Lincoln. 


\title{
Effect of an Electric Field on the Surface Tension of a Dipolar-Quadrupolar Fluid and Its Implication for Sign Preference in Droplet Nucleation
}

\author{
V. B. Warshavsky and X. C. Zeng \\ Department of Chemistry, University of Nebraska-Lincoln, Lincoln, Nebraska 68588
}

(Received 9 July 2002; published 22 November 2002)

\begin{abstract}
The effect of a uniform electric field on interfacial properties of dipolar-quadrupolar fluids is investigated by using the density-functional theory. As in the case of purely dipolar fluids the (thermodynamic) surface tension is always altered by the external field, regardless of the direction of the field. However, unlike the purely dipolar fluids, for two given external fields with the same strength but exactly opposite direction the magnitude of variation in the surface tension is different. This apparent symmetry breaking by reversing the field direction suggests a new molecular mechanism to explain the phenomenon of sign preference in droplet formation on charged condensation centers.
\end{abstract}

DOI: $10.1103 /$ PhysRevLett.89.246104

PACS numbers: 68.03.-g, 82.60.Nh, 92.60.Jq, 92.60.Ls

Ample evidences from experiments (e.g., cloud chamber experiments) and computer simulations indicate that the rate of droplet formation on charged condensation centers depends on the sign of the charge, a phenomenon known as the sign preference in ion-induced heterogeneous nucleation [1-7]. For example, water droplets nucleate faster on a negative ion (anion preference), whereas methanol droplets nucleate faster on a positive ion (cation preference), provided the magnitude of all charges are identical. A better understanding of the sign preference phenomena, therefore, has practical implications in controlled droplet production.

From a molecular point of view, the sign preference behavior can be attributed to certain asymmetry in molecular interaction. In fact, idealized molecular models such as the Stockmayer fluid which has a point dipole at the center of the Lennard-Jones particle will not show any sign preference in droplet formation because the Lennard-Jones particle is spherical. To explain the sign preference most theories invoke molecular models with explicit anisotropic steric interaction [8,9]. For these purely dipolar model fluids, marked sign preference behavior, manifested in distinctive barrier height to nucleation, was observed. Anisotropic nonpolar molecular fluids (e.g., $\mathrm{CS}_{2}$ ) may also show weak sign preference if the molecules are polarizable [10]. For dipolar systems such as hydrogen chloride $(\mathrm{HCl})$, the first molecular mechanism (due to anisotropic steric interaction) plays a major role in the sign preference whereas for nonpolar fluids the polarization mechanism dominates.

In this Letter, we present a new molecular mechanism that can be appreciable for strongly dipolar-quadrupolar (DQ) fluids to account for the sign preference in ioninduced droplet condensation. Many practical systems (e.g., water) do in fact have both a large dipole moment and also appreciable quadrupole moments. However, real dipolar/quadrupolar molecules typically have an anisotropic structure which leads to the first molecular mecha- nism. To separate the new molecular mechanism from the first one, an idealized molecular model is considered here. The molecule is a spherical Lennard-Jones particle with a point dipole as well as a point quadrupole at its center. Thus, the molecules exhibit no apparent anisotropic steric interaction. In a previous work we have shown that for purely point-dipolar fluids [11] the surface tension is not affected by reversing the direction of the external field. On the basis of the classical theory of nucleation, the barrier height to droplet formation is directly related to the surface tension. Therefore, neither purely point-dipolar nor point-quadrupolar fluids are expected to show sign preference in droplet formation because changing the sign of a charged condensation center is merely reversing the direction of external field. However, by combining a point quadrupole with the point dipole, an intrinsic asymmetry of charge distribution is introduced without invoking explicit anisotropic molecular structure.

Consider a single-component molecular fluid which consists of the particles having an electric dipole $\mu_{0}$ and quadrupole $Q$ moments. The pairwise intermolecular potential is denoted by the potential function $u\left(\vec{r}_{1}, \vec{r}_{2}\right.$, $\omega_{1}, \omega_{2}$ ), where $\vec{r}_{1}$ and $\vec{r}_{2}$ represent the position of molecules 1 and 2 , and $\omega_{1}$ and $\omega_{2}$ describe the orientation of molecular axes of molecules 1 and 2. To proceed with the density-functional theory (DFT), we divide the intermolecular potential into two parts: a strongly repulsive part (the reference potential) $u_{\text {ref }}\left(\vec{r}_{1}, \vec{r}_{2}, \omega_{1}, \omega_{2}\right)$ and a weakly attractive part (the perturbative potential) $u_{\text {per }}\left(\vec{r}_{1}, \vec{r}_{2}, \omega_{1}, \omega_{2}\right)$. In this study, the reference potential is simply chosen to be the hard-sphere potential $u_{\text {ref }}\left(r_{12}\right)=$ $+\infty$ for $r_{12} \leq d$, and $=0$ for $r_{12}>d$, where $r_{12}=$ $\left|\vec{r}_{12}\right|=\left|\vec{r}_{2}-\vec{r}_{1}\right|, d$ is the diameter of the hard sphere; $u_{\text {per }}$ is given by $\left[-4 \epsilon\left(d / r_{12}\right)^{6}+\left(u_{11}+u_{12}+u_{21}+\right.\right.$ $\left.\left.u_{22}\right)\right] H\left(r_{12}-d\right)$, where $H(r)$ is the Heaviside step function, $u_{11}$ and $u_{22}$ are the dipole-dipole and the quadrupole-quadrupole interaction, respectively, and 
$u_{12}$ and $u_{21}$ are the dipole-quadrupole interactions. In general, the multipolar potential $u_{l_{1} l_{2}}$ of linear molecules can be written as a sum of spherical harmonics $Y_{\operatorname{lm}}$ [12].

The classical DFT approach starts with the free energy of an inhomogeneous system characterized by the molecular density profile $\rho(\vec{r}, \omega)=\rho(\vec{r}) \hat{f}(\vec{r}, \omega)$. Here, $\rho(\vec{r})$ denotes the number density of molecules without specifying the molecular orientation and $\hat{f}(\vec{r}, \omega)$ denotes the distribution function of molecular orientation $\omega=$ $(\theta, \varphi)$, with $\int d \omega \hat{f}(\vec{r}, \omega)=1$. The grand potential of the system in the external field $V_{\text {ext }}(\vec{r}, \omega)$ is given by [13-15]

$$
\begin{aligned}
\Omega[\rho(\vec{r}, \omega)]= & \int d \vec{r} f_{\mathrm{hs}}[\rho(\vec{r})]+S / \beta+\Omega_{\mathrm{int}} \\
& -\int d \omega d \vec{r} \rho(\vec{r}, \omega)\left[\mu-V_{\mathrm{ext}}(\vec{r}, \omega)\right],
\end{aligned}
$$

where $\mu$ is the chemical potential, $\beta=1 / k_{B} T$ ( $k_{B}$ is the Boltzmann constant and $T$ the temperature), and $f_{\text {hs }}[\rho(\vec{r})]$ is the free-energy density of the hard-sphere reference system. The term $S=\int d \vec{r} \rho(\vec{r})\langle\ln [4 \pi \hat{f}(\vec{r}, \omega)]\rangle$, where $\langle\ldots\rangle=\int d \omega \ldots \hat{f}(\vec{r}, \omega)$, accounts for the loss of orientational entropy in the reference system. Calculation of the interaction contribution $\Omega_{\text {int }}$ which arises from the perturbative potential $u_{\text {per }}$ generally requires a certain approximation to the pair correlation function of the inhomogeneous system. An often used approximation for multipolar systems is the so-called modified meanfield (MMF) approximation $[13,14]$ which starts with the low-density approximation to the pair distribution function, followed by expanding the Boltzmann factor $e^{-\beta u_{\mathrm{per}}}$ in powers of $\beta u_{\text {per }}$, and then keeping terms up to the second order. The MMF provides a sensible DFT scheme to describe a weakly multipolar system $[13,14]$. The resulting expression for $\Omega_{\text {int }}$ is

$$
\begin{aligned}
\Omega_{\mathrm{int}}= & \frac{1}{2} \int d \vec{r}_{1} d \vec{r}_{2} d \omega_{1} d \omega_{2} \rho\left(\vec{r}_{1}, \omega_{1}\right) \rho\left(\vec{r}_{2}, \omega_{2}\right) e^{-\beta u_{\mathrm{ref}}\left(r_{12}\right)} \\
& \times\left(u_{\mathrm{per}}-\frac{\beta}{2} u_{\mathrm{per}}^{2}\right) .
\end{aligned}
$$

To describe the planar vapor-liquid interface, we consider a slab-shaped system with the $x-y$ plane in parallel with surfaces of the slab and with the uniform electric field $\vec{E}$ applied along the $z$ direction. The orientation distribution $\hat{f}(z, \omega)$ can be expressed as an expansion over the Legendre polynomials $\left\{P_{i}(\cos \theta)\right\}$ with the expansion coefficients $\left\{\eta_{i}(z)\right\}$ being the orientation order parameters, i.e., $\eta_{i}(z)=\left\langle P_{i}(\cos \theta)\right\rangle$. We consider the fluid with the weak orientation anisotropy, namely, $\mid \hat{f}-$ $1 / 4 \pi \mid \ll 1$. Taking into account all the considerations we obtain

$$
\begin{aligned}
\Omega\left[\rho(z),\left\{\eta_{i}(z)\right\}\right] / A= & \int_{-\infty}^{\infty} d z f_{\mathrm{hs}}(\rho(z))+\frac{1}{2 \beta} \int_{-\infty}^{\infty} d z \rho(z) \sum_{i=1}^{\infty}(2 i+1) \eta_{i}^{2}(z)+\frac{1}{2} \int_{-\infty}^{\infty} \int_{-\infty}^{\infty} d z_{1} d z_{2} k\left(z_{1}, z_{2}\right) \rho\left(z_{1}\right) \rho\left(z_{2}\right) \\
& -\int_{-\infty}^{\infty} d z \mu \rho(z)-\int_{-\infty}^{\infty} d z \mu_{0} E \rho(z) \eta_{1}(z)
\end{aligned}
$$

where

$$
k\left(z_{1}, z_{2}\right)=\sum_{i, k=0(k \leq i)}^{4}\left[\eta_{i}\left(z_{1}\right) \eta_{k}\left(z_{2}\right) \phi_{i k}\left(z_{12}\right)+\left(1-\delta_{i, k}\right) \eta_{k}\left(z_{1}\right) \eta_{i}\left(z_{2}\right) \phi_{i k}\left(-z_{12}\right)\right] .
$$

Here $z_{12}=z_{2}-z_{1}$ and the functions $\phi_{i k}(z)$ are polynomials of $\mu_{0}$ and $Q$ up to the fourth order. Applying the variational principle to the grand potential [Eq. (3)] with respect to $\rho\left(z_{1}\right)$ and $\left\{\eta_{i}\left(z_{1}\right)\right\}$ yields five coupled integral equations

$$
\beta \mu=\beta \mu_{\mathrm{hs}}\left(\rho\left(z_{1}\right)\right)+\frac{1}{2} \sum_{i=1}^{4}(2 i+1) \eta_{i}^{2}\left(z_{1}\right)-\beta \mu_{0} E \eta_{1}\left(z_{1}\right)+\beta \int_{-\infty}^{\infty} d z_{2} k\left(z_{1}, z_{2}\right) \rho\left(z_{2}\right),
$$

and

$$
\eta_{i}\left(z_{1}\right)=\frac{1}{3} \delta_{i, 1} \beta \mu_{0} E-\frac{1}{(2 i+1)} \beta \int_{-\infty}^{\infty} d z_{2}\left[\sum_{k=0(k \leq i)}^{4} \phi_{i k}\left(z_{12}\right) \eta_{k}\left(z_{2}\right)+\sum_{k=2(k>i)}^{4} \phi_{i k}\left(-z_{12}\right) \eta_{k}\left(z_{2}\right)\right] \rho\left(z_{2}\right) \quad(i=1, \ldots, 4)
$$

where $\mu_{\mathrm{hs}}(\rho(z))$ is the local chemical potential of the hard-sphere fluid, which can be accurately evaluated by using the Carnahan-Starling formula [16].

Equations (3)-(6) provide a working framework to calculate the interfacial properties of multipolar fluids. First, we calculate the vapor-liquid coexistence densities via solving the phase-equilibria equations: $\mu\left(\rho_{l}\right)=$ $\mu\left(\rho_{v}\right)$ and $\Omega\left(\rho_{l}\right)=\Omega\left(\rho_{v}\right)\left(\rho_{l}\right.$ and $\rho_{v}$ are the coexisting liquid and vapor densities, respectively). Here, we use the reduced quantities $\eta=(\pi / 6) \rho d^{3}, T^{*}=k_{B} T / \epsilon, E^{*}=$ $E\left(d^{3} / \epsilon\right)^{1 / 2}, \quad \mu_{0}^{*}=\mu_{0} /\left(d^{3} \epsilon\right)^{1 / 2}$, and $Q^{*}=Q /\left(d^{5} \epsilon\right)^{1 / 2}$, and we consider $\mu_{0}^{*}=1, Q^{*}=1$, and the maximum field strength $\left|E^{*}\right|=0.5$ [17]. As in the case of purely dipolar fluids [11], the external field reduces the critical temperature $T_{c}^{*}$, increasing $\eta_{v}$ but decreasing $\eta_{l}$. When 


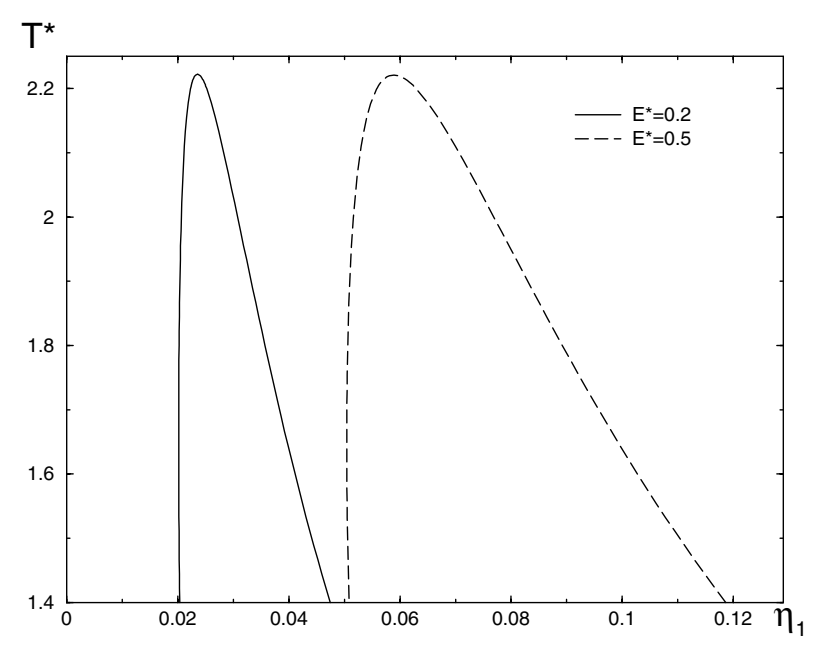

FIG. 1. The orientation order parameter $\eta_{1}$ at vapor-liquid coexistence for two given external fields. The right branch with larger values of $\eta_{1}$ corresponds to the vapor phase and the left branch corresponds to the liquid phase.

$E^{*}=0$ the four order parameters are all zero, but when $E^{*} \neq 0$ the order parameters $\eta_{1}$ and $\eta_{3}$ became nonzero. In Fig. 1 the right branch of the $T^{*}-\eta_{1}$ curve corresponds to the coexisting vapor phase whereas the left branch corresponds to the liquid phase [11]. One can see that $\eta_{1}$ is very sensitive to the strength of the external field. However, $\eta_{3}$ are about 2 orders of magnitude smaller than $\eta_{1}$. Near $T_{c}^{*}$ the mean-field scaling relations $\eta_{l}-$ $\eta_{v} \sim \tau^{1 / 2}$ and $\eta_{1 v}-\eta_{1 l} \sim \tau^{1 / 2}$ (where $\tau=1-T^{*} / T_{c}^{*}$ ) are satisfied for all the considered external field $E^{*}$.

The interfacial density profile $\eta(z)$ and the orderparameter profiles $\eta_{i}(z)(i=1-4)$ can be determined by solving the integral equations (5) and (6) using the iterative method [11]. Figures 2 and 3 display two leading order-parameter profiles $\eta_{1}(z)$ and $\eta_{2}(z)$. It is seen that when $E^{*}=0$ both $\eta_{1}^{(0)}(z)$ and $\eta_{2}^{(0)}(z)$ [superscript (0) denotes the values when $E^{*}=0$ ] are nonzero at the interface, which is a unique feature for the DQ fluids [18]. The fact that $\eta_{1}^{(0)}(z)$ is negative throughout the interfacial region indicates that molecules tend to align the dipole pointing towards the bulk liquid, while that $\eta_{2}^{(0)}(z)$ is negative on the liquid side but positive on the vapor side of the interface indicates molecules tend to orient the dipole in parallel with the interface on the liquid side but normal to the interface on the vapor side $[13,15]$. The degree of local orientational order at the interface can be described by the values $\alpha_{1}=\left|\min \left[\eta_{1}^{(0)}\right]\right|$ and $\alpha_{2}=$ $\max \left[\eta_{2}^{(0)}\right]-\min \left[\eta_{2}^{(0)}\right]$. Near $T_{c}^{*}$ the temperature scaling relations of these two quantities are given by $\alpha_{1} \sim \tau$ and $\alpha_{2} \sim \tau^{3 / 2}$. The 10-90 width of the interface $W$ scales with $\tau$ as $W \sim \tau^{-1 / 2}$.

An important feature shown in Fig. 2 is that when the external field is in the direction from the liquid to vapor (i.e., the positive field, $E^{*}>0$ ) values of $\left|\eta_{1}(z)\right|$ are always less than or equal to those of $\left|\eta_{1}(z)\right|$ if the direction of the field is reversed (i.e., the negative field, $E^{*}<0$ ).

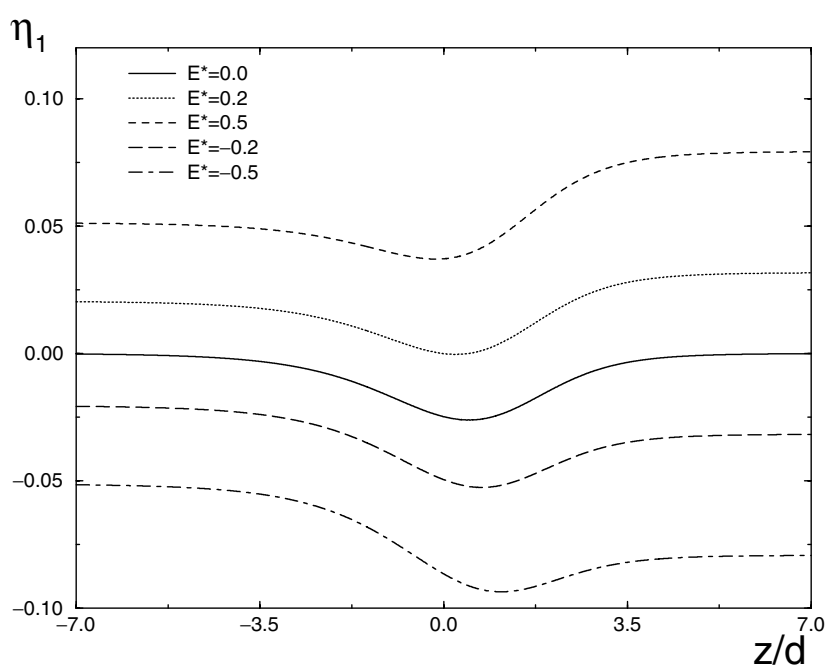

FIG. 2. Interfacial order-parameter profile $\eta_{1}(z)$ at $T^{*}=1.96$ for various given strengths and signs of external field.

Note that this "symmetry breaking" behavior due to a reverse of the field direction never occurs in purely dipolar fluids. In the DQ fluid, however, the positive field induces a local orientational ordering that is against, at the interface, the spontaneous ordering produced at the zero field. On the other hand, in the case of negative field the field-induced ordering enhances the spontaneous interfacial ordering. Indeed, as shown in Fig. 3, the positive field increases $\eta_{2}(z)$ [compared to $\eta_{2}^{(0)}(z)$ ] on the vapor side and decreases $\eta_{2}(z)$ on the liquid side. In contrast, the negative field reduces $\eta_{2}(z)$ on the vapor side and raises it on the liquid side. This contrast becomes more pronounced as the strength of the external field grows.

Finally, the surface tension $\sigma$ is determined using the thermodynamic relation $\sigma=\left(\Omega-\Omega^{\text {bulk }}\right) / A$. The reduced surface tensions $\sigma^{*}\left(=\sigma d^{2} / \epsilon\right)$ are shown in Fig. 4 as a function of $\tau$ for both $E^{*}=0$ and two fields $E^{*}=$ \pm 0.5 with the exact opposite direction. Near $T_{c}^{*}$ (or zero

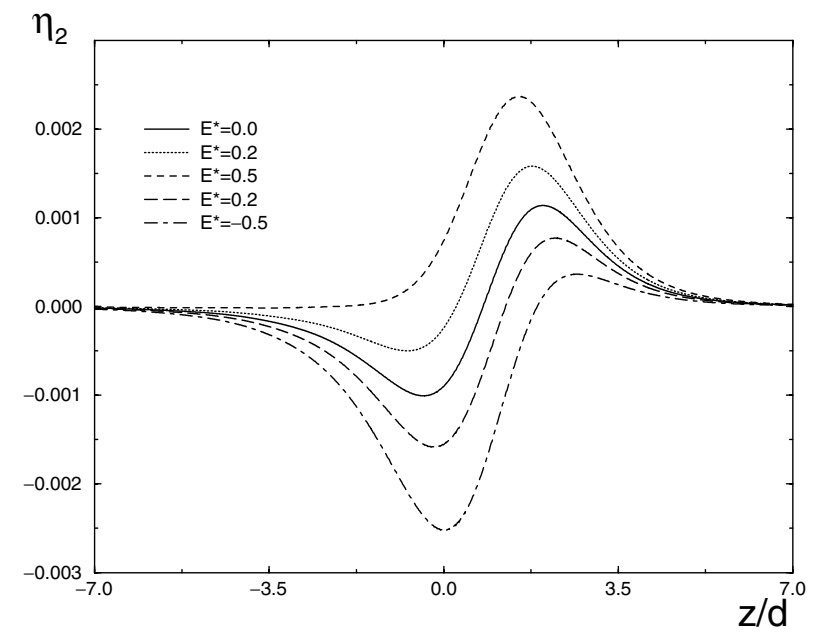

FIG. 3. Interfacial order-parameter profile $\eta_{2}(z)$ at $T^{*}=1.96$ for various given strengths and signs of external field. 


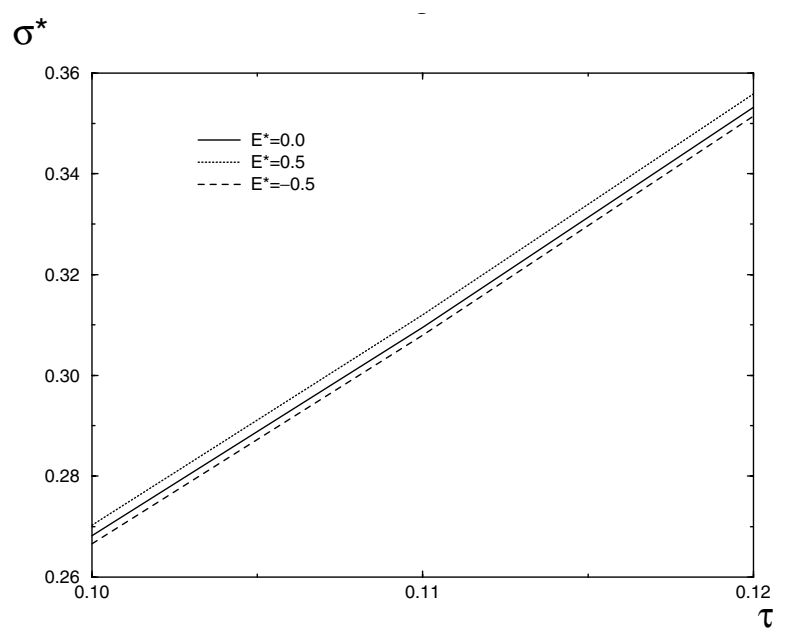

FIG. 4. Temperature dependence of the reduced surface tension $\sigma^{*}=\sigma d^{2} / \epsilon$ for zero field and two nonzero fields that are normal to the interface but with the exact opposite direction.

$\tau$ ) the mean-field scaling relation $\sigma^{*} \sim \tau^{3 / 2}$ is satisfied for both zero and nonzero fields. More interestingly, the negative field $E^{*}=-0.5$ reduces $\sigma^{*}$, whereas the positive field $E^{*}=0.5$ enhances $\sigma^{*}$. This field-direction dependence does not occur in purely dipolar fluids [11]. This symmetry breaking by reversing the field direction suggests a new molecular mechanism (without invoking explicit anisotropic molecular structure) to explain the sign preference in droplet formation on charged condensation centers. In fact, when a negative charge (anion) is introduced into a liquid droplet, the anion yields a negative field; namely, the direction of the field is from vapor to the liquid droplet. Obviously, the cation counterpart will yield an opposite positive field from the droplet to vapor. Since the anion yields a smaller surface tension than the cation counterpart (provided $Q^{*}>0$ ) the classical nucleation theory (CNT) [19] should predict that the anion will lead to a smaller barrier to the droplet formation $\left(\Delta G^{*}\right)$ than the cation counterpart [20]. Because the rate of heterogeneous nucleation is proportional to $e^{-\Delta G^{*} / k_{B} T}$, the ion-induced critical nuclei are more likely to form on negative charged particles. Consequently, the fluid will have a negative sign preference in the droplet formation on the charged condensation center. On the other hand, if $Q^{*}<0$ the DQ fluid will have a positive sign preference in droplet formation. Here, for the two given opposing fields $E^{*}= \pm 0.5$, the relative change in $\sigma^{*}$ is about $2 \%$ (Fig. 4). As a comparison, for a purely dipolar hard-sphere fluid [8] with an off-center dipole moment $\mu_{0}^{*}=1$ and a diameter $d$, the relative change in $\sigma^{*}$ is about $4 \%$ in $E^{*}= \pm 0.5$ [21]. That these changes in $\sigma^{*}$ are within the same order of magnitude indicates that the new mechanism can become competitive in contributing to the sign preference in droplet formation if the molecules have a large dipole and quadrupole moments.
This work is supported by the National Science Foundation (XCZ).

[1] C. T. R. Wilson, Philos. Trans. R. Soc. London A 189, 265 (1897).

[2] H. Rabeony and P. Mirabel, J. Phys. Chem. 91, 1815 (1987).

[3] M. Adachi, K. Okumura, and J. H. Seinfeld, J. Aerosol Sci. 23, 327 (1992).

[4] F. He and P. K. Hopke, J. Chem. Phys. 99, 9972 (1993).

[5] J. L. Katz, J. A. Fisk, and V. M. Chakarov, J. Chem. Phys. 101, 2309 (1994).

[6] D. Kane, G. M. Daly, and M. S. El-Shall, J. Phys. Chem. 99, 7867 (1995).

[7] K. J. Oh, G.T. Gao, and X. C. Zeng, Phys. Rev. Lett. 86, 5080 (2001).

[8] I. Kusaka, Z.-G. Wang, and J. H. Seinfeld, J. Chem. Phys. 102, 913 (1995).

[9] V. Talanquer and D.W. Oxtoby, J. Chem. Phys. 103, 3686 (1995).

[10] I. Kusaka, Z.-G. Wang, and J. H. Seinfeld, J. Chem. Phys. 103, 8993 (1995).

[11] V. B. Warshavsky, T.V. Bykov, and X. C. Zeng, J. Chem. Phys. 114, 504 (2001).

[12] C. G. Gray and K. E. Gubbins, The Theory of Molecular Fluids (Clarendon, Oxford, 1984).

[13] P. I. Teixeira and M. M. Telo da Gama, J. Phys. Condens. Matter 3, 111 (1991).

[14] V. Talanquer and D.W. Oxtoby, J. Chem. Phys. 99, 4670 (1993).

[15] P. Frodl and S. Dietrich, Phys. Rev. A 45, 7330 (1992).

[16] N. E. Carnahan and K. E. Starling, J. Chem. Phys. 51, 635 (1969).

[17] The chosen $\mu_{0}^{*}$ and $Q^{*}$ values are close to those of $\mathrm{HCl}$ with the reduced molecular parameters $\mu_{0}^{*}=0.903$, $Q^{*}=0.949, \quad \epsilon / k_{B}=218 \mathrm{~K}, \quad$ and $d=3.51 \AA \quad$ [see G. Stell, J.C. Rasaiah, and H. Narang, Mol. Phys. 27, 1393 (1974)]. With the $\mathrm{HCl}$ parameters, $\left|E^{*}\right|=0.5$ corresponds to an external field $E \sim 4 \times 10^{8} \mathrm{~V} / \mathrm{m}$. To compare, the field strength near a charged particle (e.g., 1e) ranges from $1.2 \times 10^{10} \mathrm{~V} / \mathrm{m}$ at the first solvation shell to $4.7 \times 10^{8} \mathrm{~V} / \mathrm{m}$ at the fifth solvation shell.

[18] B. Yang, D. E. Sullivan, B. Tjipto-Margo, and C. G. Gray, Mol. Phys. 76, 709 (1992).

[19] A. I. Rusanov and F. M. Kuni, J. Colloid Interface Sci. 100, 264 (1984).

[20] Note that according to the CNT the barrier of nucleation is controlled by both bulk and surface free energies. However, since reversing the field direction does not change the bulk free energy for point DQ fluids (nor for point dipolar fluids), the change in barrier height will stem solely from the change in $\sigma^{*}$.

[21] Here we consider the model in that the point dipole is located off-center by a distance of $0.1 d$ [V. B. Warshavsky and X.C. Zeng (to be published)]. 